\title{
Protozoan Diseases of Livestock in Arunachal Pradesh - An Overview
}

\author{
Tilling Tayo, Neeta Longjam¹ and Bangkeng Perme \\ Indian Veterinary Research Institue, \\ Izatnagar - 243122, Dist. Bareilly (UP) India. \\ 1. College of Veterinary Science, Assam Agricultural University Khanapara, Guwahati- 781022 \\ * Corresponding author email : tilling.tayo@gmail.com \\ Published Online : 30-04-2011
}

\begin{abstract}
The people (Tribes) of Arunachal Pradesh have the natural tendency to remain close contact with animals since immortal. The domestic animals are kept in basement of the house with human occupants in the first floor of same house. They remain in close contact with cattle, sheep, goat, pigs, poultry, cats and dogs throughout the year, exposing them to many animal born diseases of occupational risk. People are not aware of zoonotic diseases sharing between domesticated animal and human beings except for Rabies. So, there is regular out break of most common protozoan zoonotic diseases in human Giardiosis caused by Giardia spp. and amoebosis caused by Entamoeba spp. in the state. Another important disease of clinical significance is toxoplasmosis. Toxoplasma usually causes a febrile illness and abortions in pregnant women. The source of infection in humans may be due to close proximity with cats and unhygienic sanitation. Similarly high incidences of sarcocystis were reported form many district of Arunachal Pradesh. Source of transmission were adjudge to be eating of smoked pork and other meat product along with natural habit of eating raw vegetable as salad from contaminated areas could be the probable mode of transmission.
\end{abstract}

Keywords: Protozoa, Zoonoses, Transmission, Human, Animal, Occupational risk,

\section{I ntroduction}

Arunachal Pradesh means "land of the dawn lit mountains" in Sanskrit. It is also known as "land of the rising sun" in reference to its position as the easternmost state of India. It borders the states of Assam and Nagland to the south and southeast, and shares borders with Burma/Myanmar the east, Bhutan the northwest, and Tibet in the north. The current border is the McMahon agreed upon by Great in and the then de facto independent Tibet in a 1914 treaty.

Arunachal Pradesh with a massive $79.59 \%$ rural population is the largest state in the North-East India (2001 census). Consequently, the economy of the state is based of agriculture. Jhum cultivation and Terrace farming are the major patterns that the farmers employ to uplift agriculture in Arunachal Pradesh. In Jhum cultivation, lands are prepared by cutting down or burning the unwanted cultivation, while in terrace farming hilly cultivated lands are shaped into multiple terraces to prevent soil erosion and quick runoff of water.

\section{Climate}

The climate of Arunachal Pradesh varies with elevation. Areas that are at a very high elevation in the Upper Himalayas close to the Tibetan border enjoy an alpine or Tundra climate. Below the Upper Himalayas are the Middle Himalayas, where people experience a temperate climate. Areas at the sub-Himalayan and sea-level elevation generally experience humid, subtropical climate with hot summers and mild winters.

Arunachal Pradesh receives heavy rainfall of 80 to 160 inches $(2,000$ to $4,100 \mathrm{~mm})$ annually, most of it between May and September. The mountain slopes and hills are covered with alpine, temperate, and subtropical forests of dwarf rhododendron, oak, pine, bamboo, holong (Shorea) and teak are the main economically valuable species.

\section{Livestock in Arunachal Pradesh}

Agriculture and livestocks are the primary occupation in Arunachal Pradesh. The animals that form the bulk of the livestock are cows, mithun, yak, goats, pigs, sheep and poultry. The livestock's are not only employed solely for agricultural purpose, they also have the worth and usage value of their own. They are also the sources of milk, meat, drought, egg, manure, ritual scarification and also as a means of transportation in rural areas.

Mithun is the most important livestock domesticated animal amongst the tribes of arunachal Pradesh and it is also commonly known as ATM 
(Automated teller machine) of tribes of Arunachal Pradesh. Mithuns are widely distributed throughout the state and is concentrated mainly at the altitudes 300-3000 meters above sea level. Total mithun population (1, 84, 394 nos., Livestock census, 2003) in the state is found to be in increasing trend as revealed from the comparative of different Quinquennial years. Lower Subansiri (31,262 nos.) district has the highest mithun population amongst 16 districts in the state, whereas Upper Digang valley has lowest (1,735 nos.) mithun population. However, Changlang is the only district in Arunachal Pradesh where mithuns are not found (17th census 2003). Pig meat (pork) is liked by each and every community in the region. So, pigs are the second most important livestock animals which are reared in every house hold, maintained in kitchen waste.

Yak is the most important livestock for Brokpa (a pastoral community of Mompas tribe, whose professional and livelihoods is dependent on yak rearing and moving them for grazing from one place to another) community of Monpa tribe inhabiting in the western part of Arunachal Pradesh i. e Tawang and west kameng district (Ranjay K Singh and brokpa community, 2009), the number of yaks were reported to be 7132 and 803 respectively (17th census 2003). The state experiences a negative growth rate of buffalo, but the number of pigs and poultry farms per household in Arunachal Pradesh is maximum among all other North-eastern Indian states. Arunachal Pradesh faces many constraints in developing its livestock's resources as $76.6 \%$ of total geographically area are covered under forest. So, whatever grazing land is there, they are being fast occupied either for cultivation or urbanization. Secondly, the absence of livestock feed is posing another barrier and finally the unscientific method of livestock's rearing is making the scenario of Arunachal Pradesh livestock's more miserable. In addition to that with rapid growth of human populations increase parasitic infestation, lack of improved animal breeds and vaccination awareness are restraining the development of livestock sector in Arunachal Pradesh.

\section{A. Protozoan parasites of intestinal tract}

Coccidiosis a parasitic protozoan disease commonly causes by the members of the family Eimeriidae, which include the genera Eimeria and Isospora. Protozoan of Eimeria spp are responsible for caucusing great economic loss in livestock sector. It is the genus Eimeria that is of important concern in cattle, mithun, yak, sheep, goat and poultry.
The life cycle of these parasites is complex. Single cell oocysts (eggs) are passed in the feces of cattle, are resistant to disinfectants, and can remain in the environment (particularly moist, shady areas) for long periods of time and maintain their infectivity. The oocysts sporulate and these sporulated oocysts are ingested by the host and the sporozoites are released in the intestine. Sporozoites enter the intestinal cells, form trophozoites, which in turn divide into many merozoites. These merozoites penetrate additional intestinal epithelial cells and form more meronts. Eventually, macrogametes and microgametes are formed which combine to produce the next generation of oocysts. When the oocysts are mature, they rupture the host cell and are released into the lumen of the intestine and pass out in the feces.

The replication of the coccidia within the host's intestinal cells and the subsequent rupture of the cells are responsible for the disease and the clinical signs that develop. The severity of the disease is directly related to the dose of infective oocysts that are ingested. The more oocysts ingested, the more severe the subsequent disease. With light infections, the damage to the gut cells is minimal and because the cells in the gastrointestinal tract are replaced rapidly the damage is quickly repaired. In the case of heavy infections, about two weeks after the oocysts are ingested, most of the epithelial cells at the base of the intestinal glands are occupied by meronts or ametocytes. As these cells rupture, damage is severe and there is loss of blood into the feces. Also, fluid, electrolytes, and blood proteins (albumin) are lost. Most animals infected with coccidia do not show signs of illness. This is due to the normally low dose and after a course of infection the animal is immune to that particular Eimeria species. However, this does not mean they are immune to all Eimeria species, therefore, coccidiosis is primarily a disease of the young where there is crowding, stress, and/or nonimmune animals. Older animal certainly act as a reservoir and shed oocysts into the environment. Stress such as shipping, weaning, dietary changes, cattle shows or sales, and other problems can precipitate an outbreak of coccidiosis. The clinical signs manifested by coccidiosis are Diarrhea (bloody at times), Straining (tenesmus), Loss of appetite, Fever (slight), Debility, and Death (in severe cases). Many affected animals experience weight loss or decreased weight gains without showing obvious signs of illness and these cattle account for the majority of the economic losses.

Ruminants: Most of the ruminants (cattle, mithun, 
yak, sheep, and goat) are exposed to coccidia infection, a serious managemental disease in small ruminants and large ruminants below 6 month of age and morbidity in higher age groups, many of the infections are selflimiting and mild or asymptomatic. The parasitic infection starts increasing by spring season as environmental factors become conducive and more favourable through the summer season. Rajkhowa, et al., (2004) has however observed highest prevalence $(84.6 \%)$ during monsoon, lowest $(27.3 \%)$ in premonsoon and $41.7 \%$ in winter in mithun calves in Nagaland. Similar trends of disease prevalence were reported in Arunachal Pradesh (Annual report, 2008). The species identified from pooled faecal samples in disease investigation laboratory Nirjuli, revealed, Eimeria bovis, E. zuernii, E. bukidnonensis, E. auburnensis, E. ellipsodalis, E. Canadensis and E. cylindrica. Out of these species Eimeria zuernii and $E$. bovis were most predominant. The predominance of Eimeria bovis was earlier reported in domestic animals by Deka et al., (1995), and in mithun calves by Rajkhowa, etal., (2004).

However, sheep and goats are the main victims of coccidiosis. E. arloengi, E.intricata, E. parva and E. ninakoyakimovae. (Gupta et al., 1992; Mittra et al., 1998).

Coccidia damage the host's intestinal epithelial cells, making them more susceptible to bacterial invasion and allowing increased flow of tissue fluid into the intestinal lumen. Watery diarrhoea, which may be bloody in cattle is the major sign of infection and usually occurs about 2 weeks after ingestion of oocysts. It causes severe enteritis and diarrhoea. Clinical coccidiosis in cattle and buffalo is normally seen in animals over 4 weeks of age. Dehydration, weight loss, tenesmus, rectal prolapse, anaemia or even death may occur in some cases depending upon the species involved.

However, good stock management and avoidance of stress can considerably reduce the risk of clinical disease due to coccidiosis. Adequate nutrition, good hygiene practices, reasonable stock density and prevention of other diseases are important. Coccidiostats may be used prophylactically during the anticipated period of risk or to treat clinical cases.

Poultry: Coccidiosis is one of the most important diseases of poultry in India which account for huge economic loss. coccidia Species such as Eimeria tenella, E. necatrix, E. maxima and E. brunette, are very common in Arunachal Pradesh (Habung et al., 2008), affecting the young stock of poultry, particularly under deep litter systems. The disease is characterised by enteritis, diarrhoea and mortality. The bird develops reduced ability to absorb nutrients, which results in weight loss and eventually death. Subclinically, it is manifested by poor performance, impaired feed conversion, poor flock uniformity and poor growth. It also damages the immune system and leave poultry more vulnerable to pathogens like Clostridium, Salmonella and E. coli. The disease is considered as one of the most severe health and economical problems in poultry that causes an enormous loss to poultry producers worldwide. Medication with sulphonamides and vitamin K supplementation found to effective against Eimeria spp.

Pig: Among Eight species of Eimeria, Eimeria suis and one species of Isospora infectaions in pig-let were revelled in papum pare district (D.I.Lab., 2008). Piglets 5-15 days old are characteristically infected with only Isospora suis, which produces enteritis and diarrhea. These agents must be differentiated from viruses (swine fever), bacteria (salmonellosis), and helminth that also cause scours in neonatal pigs. Infection is characterized by a watery or greasy diarrhea, usually yellowish to white and foul smelling. A piglet appears weak, dehydrated, and undersized; weight gains are depressed, and sometimes piglets die. A contributing factor to mortality is that piglets become covered with diarrheic feces and stay damp.

\section{B. Blood Parasites of animals}

Arunachal Pradesh harbours a good numbers of blood-borne parasites compared to other north eastern states of India except Assam. This is due to the fact that the state is rich in many tick species and biting insect vectors associated with transmission of such parasites. More over the life style of livestock rearing system by trials people of Arunachal Pradesh is most commonly practiced in extensive rearing system proximity to the forest. As a matter of fact parasitic infestation is more likely to occur. Routine surveillance of blood smear from all species of animals carried out at disease investigatory laboratory (D.I.L) Nirjuli, revealed species of protozoa, ricketsia and microfilaria (nematodes).

Haemoprotozoan diseases caused by vectorborne blood protista constitute a disease entity of considerable economic importance in the state. Theileriosis, babesiosis and anaplasmosis are the most common important blood protozoan of livestocks in Arunachal Pradesh.

Anaplasmosis: Anaplasmosis is an infectious disease of livestock that causes destruction of red blood cells. The disease is caused by a rickettsial organism, belonging to the genus, Anaplasma occur in 
two forms, A. marginale and A. centrale. That is found in the red blood cells of infected cattle. Anaplasma species are transmitted either mechanically or biologically by arthropod vectors Argas persicus, Dermacentor albipictus, and Ixodes ricinus. Both the species have been recorded sporadically in lower subansiri district in cattle and mithun (Raja, 1997). A Clinical sign includes high body temperature (103$104^{\circ} \mathrm{F}$ ) associated with pyrexia with increased lacrymation and salivation in the affected animals.

The demonstration of characteristic organisms in erythrocytes is used for diagnosis. Anaplasma marginale appear as dense, rounded and deeply stained intraerythrocytic bodies, approximately $0.3-1.0 \mu \mathrm{m}$ in diameter. Most of these bodies are located on or near the margin of the erythrocyte. This feature distinguishes A. marginale from A. centrale, as in the latter most of the organisms have a more central location in the erythrocyte.

Babesiosis : Babesiosis is caused by many Babesia species that infect a wide variety of vertebrate hosts, including domestic and wild animals, as well as man. In nature, babesias typically are transmitted biologically by ixodid ticks, Boophilus microplus, but other means, such as biting flies and fomites that transfer blood from the infected carrier to a susceptible animal, may be involved in the mechanical transmission of these intra-erythrocytic parasites. Clinically it is manifested by pyrexia $\left(40-42^{\circ} \mathrm{C}\right)$ and haemoglobinurea. The disease in small ruminants has not been reported in the state. An outbreak of Babesia sp. has been reported among yak in the state (Biswas et al., 2004). The demonstration of characteristic piroplasmic stages in the erythrocytes from peripheral blood film is used for diagnosis. Berenil is drug of choice used in the region with great success in treating infected animals in addition to supportive therapy. Eradication of the Boophilus vectors would eliminate transmission of Babesiosis, over a period of time.

Theileriosis: Theileriosis caused by Theileria annulata and transmitted through the bites of Hyalomma and Rhipicephalus has been considered as the most important blood protista in the region with higher incidence in exotic breeds and the crossbred stocks of all age groups consistent with the general epidemiology of the diseases in tropical areas (Jithendran, 1998). Cases of theileriosis are generally observed during summer or rainy season when the ticks have higher activity although sporadic outbreaks have been recorded year round. The disease in mithun has been recorded in Kurung Kumey District of Arunachal Pradesh (Raja, 2007). Clinically a body temperature rise up to $107^{\circ} \mathrm{F}$, enlarged superficial lymph nodes accompanied by dullness, anorexia, salivation, lacrymation and discharge from nostrils. The demonstration of Koch's blue bodies in the lymphocytes and monocytes of the lymph node smear or peripheral blood film is pathognomonic of the disease. Tetracycline has been used with great success in treating Theileria infected animals besides the commercially available schizont vaccine for prophylactic use. Vaccination against this disease is not practised due to higher cost and non-availability. Transmission involves the tick vector and hence elimination of the vector population becomes essential for the control of theileriosis.

Trypanosomiasis: Pathogenic trypanosomes cause disease in all species of domesticated livestock throughout many of the tropical and subtropical regions of the India. Trypanosoma brucei, $T$. vivax and $T$. congolense occur wherever vector is found. The disease outbreaks have been reported from warmer plains of Pasighat district bordering Assam. However no any incidence in hilly regions of the state has been reported so far. Examination of the blood by light microscopy is the most readily applied method for diagnosis of trypanosomiasis and, more importantly, is a technique which can be easily applied in the field. The basic technique, i.e. examination of fresh or stained blood films, has been modified to improve diagnostic sensitivity by concentrating the blood through centrifugation in a haematocrit tube, namely the haematocrit centrifuge technique (HCT) or the dark ground buffy coat technique (DG) (Paris, Murray and McOdimba, 1982). The clinical signs of disease caused by these organisms vary according to the trypanosome species, the virulence of the particular isolate and the species of host infected. Acute disease is characterized by anaemia, weight loss, abortion and, if not treated, possibly death. Animals that survive are often infertile and of low productivity. In some instances, infected animals show no overt signs of disease but can succumb if stressed, for example,by work, pregnancy, milking or adverse environmental conditions (Lucking, 1988). Quinapyramine sulfate and chloride salts are used in the state with great success. No vaccine is available.

\section{Protozoan diseases of other organs}

Toxoplasmosis : Toxoplasma gondii is a world wide zoonotic disease, caused by a coccidian parasite resides in the intestine of members of the cat family. The Infection is more prevalent in stray cats acting as predators infected cats typicallly shed Toxoplasma. 
oocysts for one to two weeks and then develop immunity to the organism. However, some cats remain carriers and will shed oocysts when stressed, such as during kindling or while concurrently on steroid therapy for other disease conditions.

The infections occur in many warm-blooded animals including sheep, goats, pig, dog, and mice etc. by consuming food contaminated by Toxoplasma oocysts. Toxoplasmosis has been recognised by abortion and neonatal mortality in sheep and goats in Arunachal Pradesh. This incidence is probably due to close proximity with cat and other feline species (Rugbo, 2007). Feeding of uncooked meat should be avoided as well as proper cleaning and handling of cat litter boxes should be followed to alleviate the transmission of disease.

Sarcocystosis: There are many species of Sarcocysti affecting animal population. Generally they are tiny microscopic parasite present in muscle or intestinal cells of infected animals, group of sarcocyst often referred as cysts. Sarcocystis infection is encountered normally at the time of post-mortem examination of slaughtered animals. In most animals this parasite does not cause clinical diseases. Life cycle in definitive hosts (dogs) is initiated by eating infected slaughterhouse wastes (offal). Incidences were reported in dogs and Pigs these may be due to scavenger nature of pig (Damodar Singh, 1998). Although, few case were revealed in other species such as sheep and cattle in the state. Prophylactic administration of Amprolium or Salinomycin reduces infection. Vaccines are not available. However, infected pork could be made safe for consumption by cooking at $70^{\circ} \mathrm{C}$ for $15 \mathrm{~min}$ or by freezing at $-4^{\circ} \mathrm{C}$ for 2 days or $-20^{\circ} \mathrm{C}$ for 1 day.

\section{References}

1. Arunachal Pradesh Government department of animal husbandry and veterinary, Nirjuli. State report (2008).

2. Biswas, D., Barari, S.K. \& Pal, R.N. (1994). Ticks and lice infestations in yak. Proceedings of the first International Congress on Yak. Journal of Gansu agricultural University (Special issue, June, 1994), pp. 322-324.

3. Damodar singh, (1998). Annual report of piggery farm and disease out break, Nirjuli.

4. Deka, DK; Choudhry, S and Chakraborty, A(1995), Parasites of domestic animals and birds in Likhimpur (Assam). J.Vet. Parasitol. 9(1):21-25.

5. Government of Arunachal Pradesh department of animal husbandry and veterinary, Nirjuli. State report on 17 th quinquennial livestock census 2003 vol. 1,

6. Government of Arunachal Pradesh department of animal husbandry and veterinary, Nirjuli. Disease investigatory laboratory State report 2008.

7. Gupta, V.K., Katoch R.C., Agnihotri, R.K., Mittra, S. and Sambyal D.S (1992). Coccidiosis in Gaddi goats-a report. $J$. Hill Res., 5: 188-189.

8. Habung T, Radhe. A, Tabing. H (2008), Rearing of Vanaraja for backyard poultry farming. Unpublished.

9. Jithendran, K.P. and Sharma, A.K. (1998). A case of theileriosis in a cow: A case report. Indian Vet. Med. J., 22: 251-252.

10. Luckins, A.G. (1988). Trypanosoma evansi in Asia. Parasitol. Today, 4: 137-142.

11. Paris, J., Murray, M. \& McOdimba, F. (1982). A comparative evaluation of the parasitological techniques currently available for the diagnosis of African trypanosomiasis in cattle. Acta Trop., 39: 307-316.

12. Ranjay K Singh (2009). Indigenious knowledge of yalk breeding and management by Brokpa community in eastern Himalaya, Arunachal Pradesh. Indian journal of traditional knowledge. Vol 8 (4) pp 495-501.

13. Rajkhowa, S; Bujarbarbulah, KM; Thong Kapenlo and Rajkhow, C (2004), Prevalence of eimerian species in Mithuns of Nagaland. Indian Vet. J. 81(5): 573-574. 